\title{
Combustion Dynamics of Swirling Turbulent Flames
}

\author{
Suresh Menon, Vaidyanathan Sankaran, and Christopher Stone \\ School of Aerospace Engineering \\ Georgia Institute of Technology \\ Atlanta, Georgia 30332 \\ suresh.menon@aerospace.gatech.edu
}

\begin{abstract}
A generalized Large-Eddy Simulation (LES) methodology has been developed to simulate premixed and non-premixed gas-phase and two-phase combustion in complex flows such as those typically encountered in gas-turbine combustors. This formulation allows the study and analysis of the fundamental physics involved in such flows, i.e., vortex/flame interaction, combustion dynamics and stability, fuel-air mixing, droplet vaporization, and other aspects of combustion. Results for swirling premixed combustion undergoing combustion instability and for swirling spray combustion in full-scale gas turbine engines are discussed here. Results show that swirl can stabilize combustion in premixed system and can reduce the magnitude of the amplitude of the pressure oscillation. In two-phase systems, significant modification to the high shear regions due to vaporization of droplets is observed. Droplets are also seen to concentrate in regions of low vorticity and when they vaporize, the gaseous fuel gets entrained into regions of high vorticity. This process plays a major role in fuel-air mixing and combustion processes in two-phase systems.
\end{abstract}

\section{Introduction}

The simulation of compressible, swirling, turbulent reacting flows such as those found in contemporary power generation and aircraft gas turbine systems pose a great challenge due to the widely varying time and length scales that must be resolved for accurate prediction. In addition to the difficulty in resolving all the important turbulent scales, the presence of multiple modes/regimes of combustion and the interaction between various chemical species and physical phases (liquid and gas) in the same combustion device further complicates the modeling requirements. Here, a methodology based on Large-Eddy Simulations (LES) is developed and applied to these types of problems.

In LES, scales larger than the grid scale are computed using a time- and space-accurate scheme while the effects of the smaller, unresolved scales (assumed to be mostly isotropic) are modeled. For momentum transport closure simple eddy viscosity based sub-grid models are sufficient since the unresolved small-scales primarily provide dissipation for the energy transferred from the 
large scales. However, combustion occurs at the molecular scales and the interaction between the small-scales of motion and molecular diffusion play a major role in combustion and heat release. Thus, to properly account for heat release effects, the small scale processes must be simulated accurately (which is in conflict with the eddy viscosity approach used for momentum closure). In order to deal with these distinctly different modeling requirements, a sub-grid combustion model has been developed [1] that resides within each LES cell and accounts for the interaction between the small-scale mixing and reaction-diffusion processes.

Earlier studies 1234 have established the ability of the LES model in premixed and non-premixed systems. In the present study, the dynamics of swirling premixed flames in a gas turbine combustor is studied using the same LES approach. For two-phase reacting flow, this LES approach was extended [5] and used within a zero-Mach number (incompressible) formulation to study spray transport and vaporization in spatial mixing layers. In the present study, the two-phase model has been implemented within the compressible LES model developed earlier [24] for gas phase combustion and then used to study spray combustion in a high Reynolds number swirl flow in a gas turbine combustor.

\section{Large-Eddy Simulation Model}

The Favre filtered mass, momentum, energy, and species conservation equations are solved in the LES approach. In addition, the unresolved turbulence kinetic energy is modeled with a single sub-grid kinetic energy equation, $k^{s g s}[2$. The subgrid kinetic energy is used to close the unresolved stresses and energy/species flux terms resulting from the filtering operations. For premixed combustion studies, a thin-flame model [2] is employed while a Lagrangian droplet tracking method [6] is used to explicitly track the droplets in the Eulerian gas field in the spray simulations. In this method, the liquid droplets are tracked with a Lagrangian approach to explicitly compute the mass, momentum, energy and species transfer between the continuum and dispersed phase. The gas phase LES velocity fields and the sub-grid kinetic energy are used to estimate the instantaneous gas velocity at the droplet location. Drag effects due to the droplets on the gas phase is explicitly included. Heat transfer from gas phase to the liquid phase aids in the vaporization and the subsequent mass transfer to the gas phase. This provides the thermal coupling between the two phase. Thus, full coupling is achieved between the two phases in the simulation.

The governing equations mentioned above have been withheld for brevity; however, they along with further details can be found elsewhere [2.5]].

\section{Numerical Methodology}

The LES equations of motion are solved on a three dimensional, boundaryconforming, grid using an explicit finite-volume scheme that is fourth-order accurate in space and second-order accurate in time. No-slip, adiabatic wall con- 
ditions conditions are used along with non-reflecting inflow/out-flow boundary conditions $[7$.

The configuration used for both premixed and two-phase combustion studies consists of an inlet pipe expanding into the larger cylindrical combustion chamber. A swirling velocity profile with a swirl number of 0.56 is imposed at the inlet boundary. The mean inlet mass flow rate, temperature, and pressure are 0.435 Kilograms/second, 673 Kelvin, and 11.8 atmospheres, respectively. The Reynolds number based on inlet bulk velocity and inlet diameter is 330,000. An inflow turbulent field is generated by using a specified turbulence intensity (7\%) on a randomly generated Gaussian field.

For two-phase (spray) LES, a dilute spray is introduced at the inlet plane using $20 \mu \mathrm{m}$ droplets (future studies will incorporate a log-normal size distribution). The Stokes number, the ratio of droplet to flow time scales, is approximately 8.2. Droplets below a cut-off size of $5 \mu \mathrm{m}$ are assumed to instantly vaporize and mix. Gas phase velocities at the particle locations are interpolated using a fourth-order scheme. The governing Lagrangian (two-phase) equations are integrated with a fourth-order Runge-Katta scheme. Elastic collisions are assumed for particle/wall interaction.

A grid resolution of $141 \times 65 \times 81$ is employed for both the premixed and twophase LES. Clustering of the grid in regions of high shear is used. For the spray simulations, 120,000 droplet groups are tracked in the computational domain.

The LES solver is implemented on massively parallel systems using domain decomposition and standard Message-Passing Interface (MPI) libraries. The parallel algorithm exhibits good scalability (85\% parallel efficiency on 128 CPU's) on several high-performance computing platforms. Simulations on the Cray T3E900 typically require 900 and 3400 CPU hours for a single flow-through (the time for a fluid element to traverse the entire computational domain) for the premixed and spray calculations, respectively. In general, 5 to 10 flow-through-times are simulated for statistical analysis. The memory requirements for the premixed and spray computations are 2.9 and 12.3 Gigabytes, respectively.

\section{Combustion Dynamics in a Premixed System}

Accurate prediction of the coupling between unsteady heat release and pressure oscillation is critical to simulate combustion dynamics in dump combustors. However, proper resolution of this coupling is especially difficult due to the unsteadiness of the controlling processes (e.g., fuel injection) and the high nonlinearity of the interactions between turbulent mixing, acoustic wave motion, and unsteady heat release. Large-scale structures in the flow play a key role in the coupling process by controlling the fuel-air mixing. In non-swirling flows, axisymmetric coherent vortices are shed at the dump plane and these structures interact with the acoustic waves and heat release in the combustor. In a highly swirling flow, azimuthal (helical) instability modes are present and the interaction between the modes of motion is more complicated. In fact, swirl can stabilize or even destabilize instability in sudden expansion flows [8]. Therefore, the identification 
of flow or system parameters that control swirl induced instability is extremely important for the design of stable combustion systems.

The effect of swirl on lean premixed flames is investigated in this study. Although lean burning systems have some significant advantages (such as reduced pollutant emission and increased fuel efficiency), lean operation is sensitive to small fluctuations which under certain conditions can get amplified resulting in high-amplitude pressure oscillations. This phenomenon is often called combustion instability and understanding this phenomenon is the focus of the current study.

Two simulations are conducted in order to observe the effects of heat release on the dynamics of combustion in the swirling combustor. Case A simulates a passive flame (zero heat-release) while case B includes heat-release with a flame temperature of 1807 Kelvin. In the limit of zero heat release, the thin-flame field does not effect the flow and therefore, acts as a passive scalar that is advected by the fluid flow without affecting the flow field. In the heat release case, the flame responds to the flow field that is modified due to heat release and thermal expansion effects.

The mean and fluctuating axial and radial velocity profiles across the diameter of the combustor (at the center plane) are shown in Fig. 1 The profiles are shown at a non-dimensional distance of $\mathrm{X} / \mathrm{D}_{0}=0.2\left(D_{0}\right.$ is the diameter of the inlet pipe) downstream of the dump plane. Near the centerline, the mean axial velocity seems to be reduced due to heat release while the radial velocity is increased. Assuming a conical flame, the flame-normal expansion will be predominantly aligned in the radial direction. This divergence (broadening of the streamlines) would cause the reduction in the mean axial velocity inside the flame region and the corresponding increase in the radial component. Additionally, the magnitude of recirculation after the sudden expansion is reduced in the presence of heat release.

Root-Mean-Square $(R M S)$ velocity profiles at the same axial location are shown in Figs. $1(\mathrm{~b}, \mathrm{~d})$. In the shear layer region, $\mathrm{R} / \mathrm{D}_{0} \approx 0.5$, both simulations show high fluctuations; however, the inner core region has some distinct differences. Similar to its mean counterpart, the fluctuating axial velocity is reduced near the centerline. The other two components show the same trend of lower velocity fluctuations associated with heat release. This reduction is caused by the increased viscous dissipation in the hot products.

Shown in Fig. 2 are the pressure fluctuation spectra for the two cases. The pressure signals were recorded at the base of the dump plane where the vorticity is low. Both simulations reveal a dominant frequency (plus a harmonic) at a Strouhal number (defined as $f \mathrm{D}_{0} / \mathrm{U}_{0}$ ) of 0.88 for the cold flow and 1.12 for the reacting flow (with harmonics at 1.76 and 2.24, respectively). Analysis of the pressure amplitudes and phase angles of these pressure signals along the longitudinal axis of the combustor indicated a $3 / 4$ wave with a wave length proportional to the combustor length (from dump plane to diffuser). This wave shape is acoustic as indicated by the frequency shift from a cold flow (Case A) 


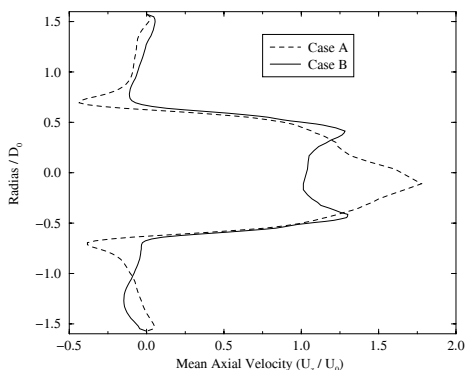

(a) $\mathrm{U}_{x}$

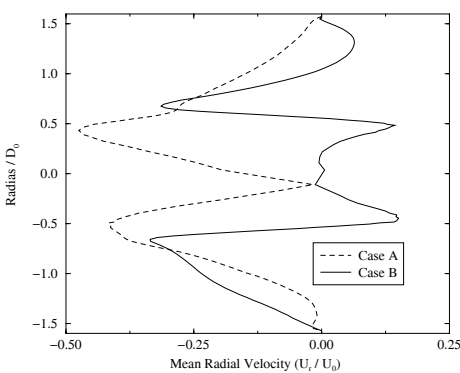

(c) $\mathrm{U}_{r}$

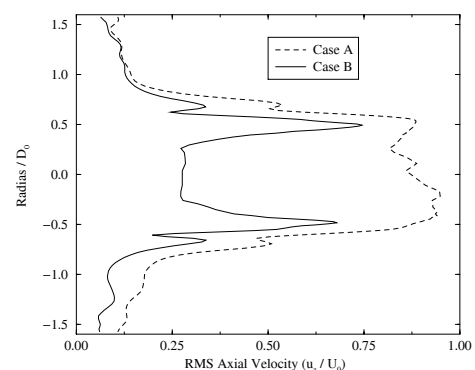

(b) $\mathrm{u}_{x}$

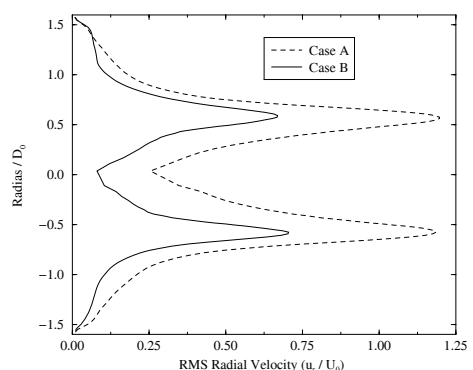

(d) $\mathrm{u}_{r}$

Fig. 1. Mean and RMS velocity profiles at $\mathrm{X} / \mathrm{D}_{0}=0.2$ downstream of the dump plane. $(\mathrm{a}, \mathrm{b})$ : Axial $\left(\mathrm{U}_{x}, \mathrm{u}_{x}\right)$ and $(\mathrm{c}, \mathrm{d}) \operatorname{radial}\left(\mathrm{U}_{r}, \mathrm{u}_{r}\right)$.

to the hot (Case B) (i.e., the frequency shift corresponds to the change in the speed of sound at the higher temperature).

As with the $R M S$ velocity profiles, Fig. 2(a) gives credence to the attenuation effect of heat release. While the acoustic wave shape is the same, the amplitude is attenuated by almost $700 \%$. A time segment of the global Rayleigh parameter $(\bar{R}(t))[9]$ (not to scale) is given in Fig. 2(b). Positive $\bar{R}(t)$ corresponds to amplification while negative indicates neutral oscillations or attenuation. This time sequence shows stable operation $(\bar{R}(t)$ is nearly always $(-))$, i.e. the pressure, $p^{\prime}$, and heat release, $\Delta q^{\prime}$, fluctuations are not in phase. Only at the higher harmonic of the pressure signal $(\mathrm{St}=2.24)$ does amplification occur, indicating that the heat release oscillations are at or near the higher frequency.

Figure 3 shows a mean and an instantaneous view of the three-dimensional flame surface. All pictures were taken with the same frame of references to allow direct comparison of the flame dimensions. Due to heat release and the associated thermal expansion, the mean flame surface is repelled (expands) outward and is 

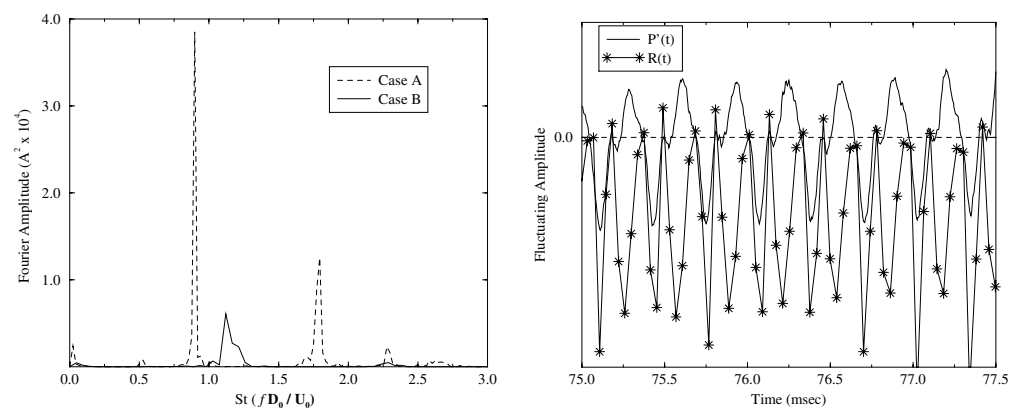

Fig. 2. (a) Fourier transform of pressure fluctuations in the combustor. (b) Time sequence of the volume averaged Rayleigh parameter, $R(t)$.

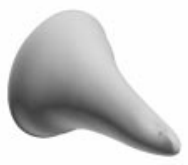

(a)

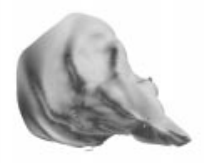

(b)

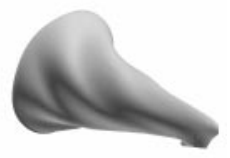

(c)

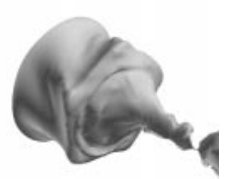

(d)

Fig. 3. Mean and instantaneous 3D flame surface: Case A (a) mean and (b) instantaneous, and Case B (c) mean and (d) instantaneous. Flow direction direction is from top left to bottom right.

also longer. However, no significant visual distinction can be made between the cold and hot instantaneous flame fronts. Both show wrinkling/elongation in the azimuthal direction (rib-shaped structures).

The rib-shaped structures in the flame front are aligned with vortex tubes generated in the swirling boundary layer that is shed from at the dump plane. An example of the vortex shedding from Case B is shown in Fig. 4(a) \& (b). Fig. 廿1a) shows the azimuthal ( $\omega_{\theta}$, dark gray) and axial $\left(\omega_{x}\right.$, light gray) vorticity. The large-scale, ring structures are predominantly $\omega_{\theta}$. Braid structures (mostly $\left.\omega_{x}\right)$ are observed in the region between the shed vortices. As the ring vortices shed, they entrain the flame and carry it along (shown in Fig. 4(b)). The flame is drawn outwards till the vortex breaks down. This vortex-flame interaction forms a flame oscillation cycle with a time-scale proportional to the vortex shedding rate. The shedding rate is strongly coupled with the longitudinal acoustic waves 


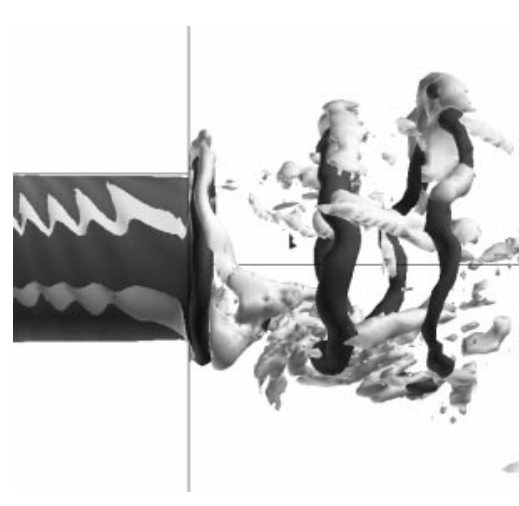

(a)

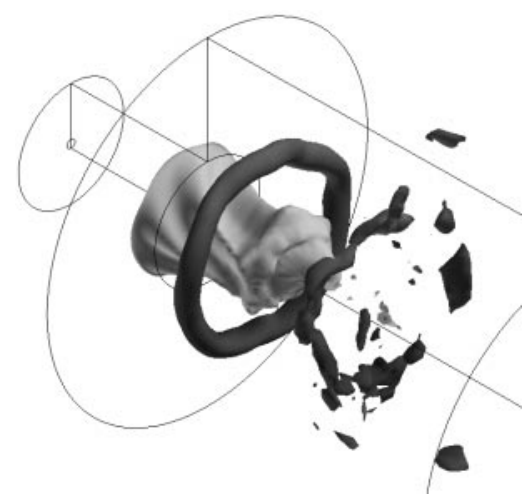

(b)

Fig. 4. Vortex-Flame interactions in the combustor (instantaneous views): (a) Tangential Vorticity, $\omega_{\theta}$ (dark gray) and Axial Vorticity $\omega_{x}$ (light gray), (b) Flame surface, $G$, (light gray) and Tangential Vorticity, $\omega_{\theta}$ (dark gray). Flow direction is from top left to bottom right in (b).

in the combustor (left running waves trip the unstable boundary layer at the dump plane causing vortex roll-up).

\section{Spray Combustion}

Three simulations are discussed here. A non-reacting gas-phase-only case, a nonreacting two-phase case (i.e., only momentum coupling) and a reacting (using a global infinite-rate kinetics) two-phase case. In all three cases, the same swirling inflow was employed. A general observation, for all three cases, shows that the heavy particles do not follow the gas phase due to their larger inertia; however, as they get smaller, due to vaporization, they equilibrate with the gas phase. Smaller droplets are observed in the recirculation bubble near the dump plane. On the other hand, in the momentum coupled case, fewer particles are seen in the recirculation bubble due to the large Stokes number associated with the particles. Large dispersion of droplets towards the outer region of the combustor (not shown) is observed. This radial spread is seen to increase with downstream distance. Larger particles that reach the wall bounce back and move downstream. Further downstream, the distribution of the droplets tends to be more uniform.

Figure 5 (a) shows the ISO-surface of the vorticity and droplet distribution in the combustor. Coherent vortex structures which are seen near the dump plane in the gas-phase simulations are quickly disintegrated in the presence of droplets. Analysis shows that droplets tend to accumulate in regions of low vorticity. This 


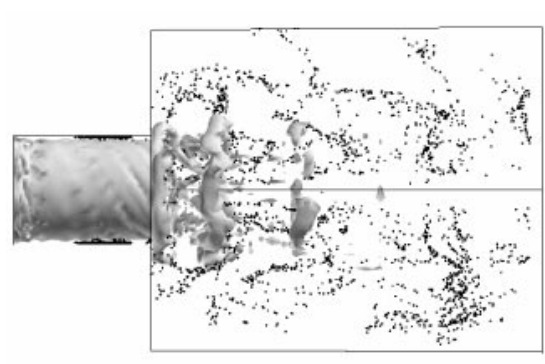

(a)

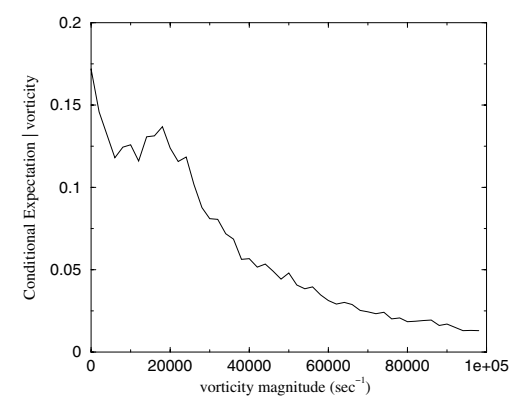

(b)

Fig. 5. (a) Contours of Vorticity ISO-surface $\left(45,000 \mathrm{~s}^{-1}\right.$ (yellow) and the droplet number distribution and (b) Conditional expectation of droplet number density.

type of preferential accumulation of droplets in regions of low vorticity was also observed in earlier studies of simpler shear flows [5. Conditional expectation of droplet number density conditioned on vorticity is shown in Fig. 5(b). The abscissa in this plot is the normalized by the vorticity magnitude. As can be seen, the probability density function is asymmetric and is biased towards low vorticity.

Figures 6(a) and (c) show respectively, the mean gas-phase velocity profiles in the stream-wise and transverse directions. These radial profiles are shown at a non-dimensional distance of 0.14 (which is slightly downstream of the dump plane). As was observed in the premixed case, the mean axial velocity is reduced with heat release. In addition, the presence of particles (with or without heat release) reduces the mean velocity, especially in the shear layer region. Thus, particle drag effects reduces large radial variation in the velocity profiles while heat release (and the associated thermal expansion) further smooths out radial gradients.

Further analysis shows that the swirl has been significantly attenuated due to the presence of the droplets. It should be noted that for flows with a large droplet to gas-phase density ratio and droplet sizes smaller than the Kolmogorov scale, the particle paths, the relative velocities (between the two phases), and the particle drag are all uniquely determined by the Stokes number. Therefore, future studies at different Stokes numbers and more realistic droplet size distributions are needed.

Figures 6 (b) and (d) show respectively, the root mean square velocity fluctuations in the stream-wise and transverse directions. It can be seen that the turbulent fluctuations have been attenuated in the presence of the particles. The presence of droplets decreases the turbulence level by introducing additional dissipation. In particular, turbulent fluctuations have been attenuated significantly 


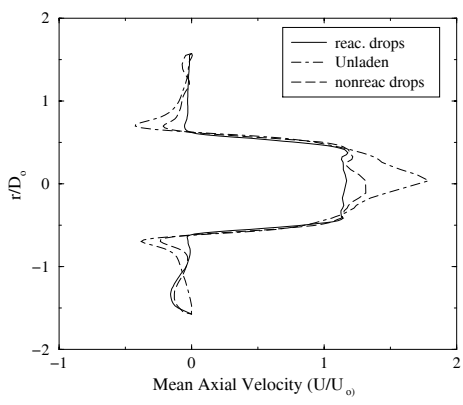

(a)

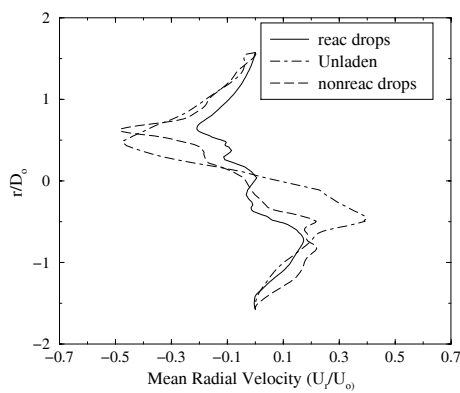

(c)

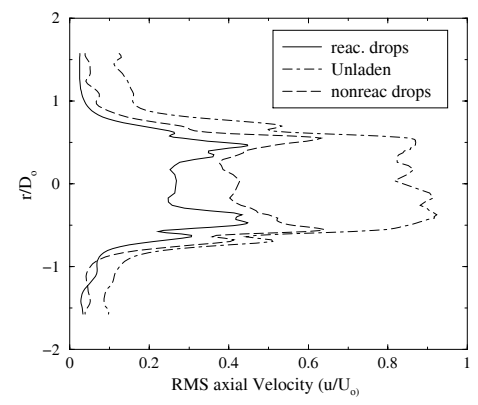

(b)

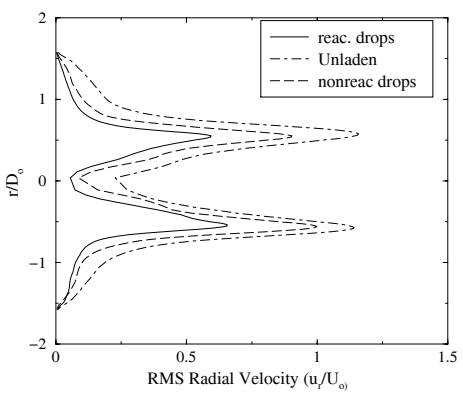

(d)

Fig. 6. Mean and RMS velocity profiles at $X / D_{0}=0.14$ downstream of the dump plane. $(\mathrm{a}, \mathrm{b})$ : Axial $\left(\mathrm{U}_{x}, \mathrm{u}_{x}\right)$ and $(\mathrm{c}, \mathrm{d}) \operatorname{radial}\left(\mathrm{U}_{r}, \mathrm{u}_{r}\right)$.

in regions where turbulent intensities are high in unladen flows (i.e., in regions of high shear). This is because in these regions, the local Stokes number based on the turbulent time scales is high leading to increased attenuation of the turbulence closer to the shear layer where the turbulence production is very high. This observation is consistent with an earlier study [10]. Turbulence levels in the recirculating zones are not affected significantly, due to the presence of fewer particles there.

\section{Conclusions}

Simulation of high Re, swirling premixed and spray flames in full-scale gas turbine combustors have been carried out using the same LES solver. This LES approach includes a more fundamental treatment of the interaction between the 
flame, gas-phase, and liquid flow dynamics. Combustion dynamics in the lean premixed system has been simulated and results show that the dominant mode shape is the three-quarter acoustic wave shape in the combustor. Results also show that swirl and heat release effects can stabilize the system by reducing the amplitude of pressure oscillation. Simulation of spray combustion show that many global features such as the preferential concentration of droplets in low vorticity regions, droplet dispersion and turbulence modification by the particles are all captured reasonably well. However, many other issues such as the effect of the mass loading ratio, droplet vaporization rate, and Stokes number on the turbulent reacting flow needs to be studied further. These issues are currently being addressed and will be reported in the near future.

\section{Acknowledgments}

This work was supported in part by the Army Research Office (ARO) under the Multidisciplinary University Research Initiative (MURI) and General Electric Power Systems. Computational time was provided by DOD High Performance Computing Centers at NAVO (MS), SMDC (AL), WPAFB (OH), and ERDC (MS) under ARO and WPAFB HPC Grand Challenge Projects.

\section{References}

1. V.K. Chakravarthy and S. Menon, "Large-eddy simulations of turbulent premixed flames in the flamelet regime," Combustion Science and Technology, vol. 162, pp. 1-48, 2001, to appear.

2. W.-W. Kim, S. Menon, and H. C. Mongia, "Large eddy simulations of a gas turbine combustor flow," Combustion Science and Technology, vol. 143, pp. 25-62, 1999.

3. W.-W. Kim and S. Menon, "Numerical modeling of fuel/air mixing in a dry low-emission premixer," in Recent Advances in DNS and LES, Doyle Knight and Leonidas Sakell, Eds. Kluwer Academic Press, 1999.

4. W.-W. Kim and S. Menon, "Numerical modeling of turbulent premixed flames in the thin-reaction-zones regime," Combustion Science and Technology, vol. 160, pp. 110-150, 2000.

5. S. Pannala and S. Menon, "Large eddy simulations of two-phase turbulent flows," AIAA 98-0163, 36th AIAA Aerospace Sciences Meeting, 1998.

6. J. C. Oefelein and V. Yang, "Analysis of transcritical spray phenomena in turbulent mixing layers," AIAA 96-0085, 34th AIAA Aerospace Sciences Meeting, 1996.

7. T.J. Poinsot and S.K. Lele, "Boundary conditions for direct simulations of compressible viscous flow," Journal of Computational Physics, vol. 101, pp. 104-129, 1992.

8. S. Sivasegaram and J.H. Whitelaw, "The influence of swirl on oscillations in ducted premixed flames.," Combustion Science and Technology, vol. 85, 1991.

9. S. Menon, "Active combustion control in a ramjet using large-eddy simulations," Combustion Science and Technology, vol. 84, pp. 51-79, 1992.

10. J. R. Fessler and Eaton J. K., "Turbulence modification by particles in a backward facing step flow," Journal of Fluid Mechanics, vol. 394, pp. 97-117, 1999. 